\title{
Avaliando o processo de trabalho das equipes NASF microrregião de Aquidauana, MS: Projeto Terapêutico Singular
}

\section{Evaluating the work process of NASF microrregião de Aquidauana, MS teams: Singular Therapeutic Project}

Avaliando el proceso de trabajo de los equipos NASF microrregión de Aquidauana, MS: Proyecto Terapéutico Singular

Bruna Alves de Jesus ${ }^{1}$ Karla de Toledo Candido Muller ${ }^{2}$ Alexandre Alves Machado ${ }^{3}$ Cristiano Marcelo Espinola Carvalho ${ }^{4}$

${ }^{1}$ Mestre em Biotecnologia pela Universidade Católica Dom Bosco (UCDB), Pós-graduanda em Neonatologia e Pediatria (Famper). Enfermeira pela UCDB. Universidade Católica Dom Bosco, Campo Grande, MS. E-mail: brunaalvesdejesus@hotmail.com

${ }^{2}$ Doutora em Saúde e Desenvolvimento na Região Centro-Oeste Universidade Federal de Mato Grosso do Sul (UFMS). Professora Titular da Universidade Católica Dom Bosco, Campo Grande, MS. E-mail:karla@ucdb.br

${ }^{3}$ Doutor em Ciências Farmacêuticas (USP). Pesquisador colaborador Universidade Federal de Mato Grosso do Sul (UFMS). Universidade Católica Dom Bosco, Campo Grande, MS. E-mail: amachado.net@gmail.com

${ }^{4}$ Doutor em Biologia Celular e Molecular pela Fundação Oswaldo Cruz. Pró-Reitor de Pesquisa e Pós-Graduação da Universidade Católica Dom Bosco.

E-mail: cristiano@ucdb.br 
Resumo: O objetivo principal deste estudo foi avaliar a utilização da ferramenta tecnológica de apoio Projeto Terapêutico Singular (PTS) pelas equipes Núcleo de Apoio à Saúde da Família (NASF) da microrregião de Aquidauana, localizado no Estado de Mato Grosso do Sul. Trata-se de um estudo de caráter quantitativo-descritivo, no qual foram entrevistados 15 profissionais de diferentes cidades da microrregião de saúde de Aquidauana, em Mato Grosso do Sul, e extraídas as ideias centrais com foco no conhecimento sobre a ferramenta PTS. Oito entrevistados demonstraram entendimento sobre como utilizar a ferramenta PTS e aplicabilidade a respeito do recurso terapêutico, e sete não tinham contato e não sabiam a finalidade do recurso. Portanto o PTS deve ser valorizado no que se trata promoção a saúde, já que produz saúde através dos impactos gerados de suas ações que atuam diretamente sobre os condicionantes e determinantes sociais da saúde da população; nesse contexto a gestão dos serviços é indubitavelmente necessária para o funcionamento do NASF.

Palavras-chave: saúde pública; promoção da saúde; tecnologia em saúde.

\begin{abstract}
The main objective of this study was to evaluate the use of the technological tool to support the Unique Therapeutic Project (PTS) by the NASF teams of the Aquidauana microregion, located in the State of Mato Grosso do Sul. This is a quantitative-descriptive study, where 15 professionals from different cities of the Aquidauana health microregion in Mato Grosso do Sul were interviewed and the central ideas focused on the knowledge about the PTS tool were extracted. Eight interviewees demonstrated an understanding of how to use the PTS tool and applicability regarding the therapeutic resource, and seven had no contact and did not know the purpose of the resource. Therefore, PTS must be valued in what concerns health promotion, since it produces health through the impacts generated from its actions that act directly on the determinants and social determinants of the health of the population, in this context the management of services is undoubtedly necessary for the functioning of the NASF.
\end{abstract}

Keyword: public health; health promotion; technology in health.

Resumen: El objetivo principal de este estudio fue evaluar la utilización de la herramienta tecnológica de apoyo Proyecto Terapéutico Singular (PTS) por los equipos NASF de la microrregión de Aquidauana, ubicada en el Estado de Mato Grosso do Sul. Se trata de un estudio de carácter cuantitativo-descriptivo, donde fueron entrevistados 15 profesionales de diferentes ciudades de la microrregión de salud de Aquidauana, en Mato Grosso do Sul y extraídas las ideas centrales con foco en el conocimiento sobre la herramienta PTS. Ocho entrevistados demostraron entendimiento sobre cómo utilizar la herramienta PTS y aplicabilidad respecto del recurso terapéutico, y siete no tenían contacto y no sabían la finalidad del recurso. Por lo tanto el PTS debe ser valorado en lo que se trata promoción la salud, ya que produce salud a través de los impactos generados de sus acciones que actúan directamente sobre los condicionantes y determinantes sociales de la salud de la población, en ese contexto la gestión de los servicios es indudablemente necesaria para el desarrollo funcionamiento del NASF.

Palabras clave: salud pública; promoción de la salud; tecnología en salud. 
Avaliando o processo de trabalho das equipes NASF microrregião de Aquidauana, MS: Projeto Terapêutico Singular

\section{INTRODUÇÃO}

Os Núcleos de Apoio à Saúde da Família (NASF) surgiram a partir da criação da Portaria n. 154, de 24 de janeiro de 2008 (BRASIL, 2008), na intenção de criar um suporte qualificado às equipes da Estratégia Saúde da Família (ESF) na condução de situações que necessitem de outros conhecimentos e outros saberes profissionais, garantindo um cuidado mais integral. Desde então, o NASF vem sendo instalado nos municípios do Brasil, como uma estrutura especĺfica unindo profissionais de diferentes áreas de conhecimento.

No I Encontro Sul-Mato-Grossense do NASF, realizado na cidade de Aquidauana, em agosto de 2011, diversas dúvidas e questionamentos surgiram acerca de operacionalização de suas premissas e do processo de trabalho das equipes. Houve a participação maciça dos profissionais dos NASF do Estado, os quais colocaram as dificuldades enfrentadas no cotidiano dos serviços e o sentimento de impotência diante de demandas para as quais não foram formados ou capacitados para atender.

Mediante as dificuldades vivenciadas para a implantação de um novo modelo de atenção à saúde, constatou-se a necessidade do estudo sobre a realidade das práticas profissionais nos NASF no Mato Grosso do Sul. Assim, ações concretas e resolutivas poderão ser planejadas e executadas na busca da qualificação dos profissionais para a atenção à saúde dos indivíduos e coletividades no contexto em que os NASF foram idealizados.

\section{NASF E A FERRAMENTA TECNOLÓGIGA PTS}

O NASF é uma estratégia inovadora criada pelo Ministério da Saúde, em 2008, constituída de equipe multiprofissional que possui o intuito de apoiar as equipes da ESF na execução de suas ações e aumentando a abrangência das ações na atenção básica, de forma que alcance a resolubilidade e qualidade das ações programáticas (BRASIL, 2010).

O NASF vem modificando o processo de trabalho e a produção de cuidado que é oferecido à ESF, e colaborando para a implementação de ações qualificadas, porém essa estratégia ainda está em andamento para a consolidação. E as formas de organização do trabalho também são recentes e não apresentam um processo sistematizado. (GONÇALVES, 2015, p. 61) 
Contudo a organização do trabalho entre NASF e as equipes da atenção primária da ESF utilizam da corresponsabilização e de ações compartilhadas que, a partir das demandas territoriais, são desenvolvidas e planejadas entre si (BARROS et al., 2015, p. 2848).

Segundo Panizzi et al. (2017, p. 156), "trata-se de um dispositivo que tem como perspectiva a mudança no modo de produzir o cuidado, potencialmente dispara um processo de reestruturação produtiva". E essa mudança pode ser descrita como modelo tecnoassistencial, tal como assim apresentada:

A denominação de "modelo tecnoassistencial" é utilizada por Merhy para designar um processo composto por "tecnologias do trabalho em saúde" e a assistência, como uma "tecnologia do cuidado" operada por três tipos de arranjos tecnológicos: tecnologias leves, leve-duras e duras. Neste sentido, é preciso investir nas tecnologias leves do tipo relacionais, centradas nas necessidades dos usuários, invertendo o investimento nas tecnologias duras ou leve-duras, traduzidas nas normas, equipamentos e materiais. (MERHY, 2002 apud FERTONANI et al., 2015, p. 1871).

O Projeto Terapêutico Singular (PTS) é uma ferramenta tecnológica de apoio à atenção e implica a organização e desenvolvimento do processo de trabalho buscando um conjunto de propostas e condutas em que os profissionais se reúnem em prol do sujeito com um olhar holístico, unindo terapêuticas articuladas, para sujeitos individuais ou coletivos, o que se processa em quatro momentos: diagnóstico, definição de metas, divisão de responsabilidades e reavaliação, de modo que designa o movimento de coprodução e cogestão do cuidado (NASCIMENTO; OLIVEIRA, 2010). O Ministério da Saúde (BRASIL, 2010, p. 28) aponta o PTS como "um momento de toda a equipe em que todas as opiniões são importantes para ajudar a entender o sujeito com alguma demanda de cuidado em saúde e, consequentemente, para definição de propostas de ações".

Segundo Mororó (2010), Merhy e Franco (2003) entendem, o projeto terapêutico é um conjunto de condutas terapêuticas articuladas, resultantes de discussão coletiva de equipe interdisciplinar, que responda às demandas objetivas e subjetivas dos usuários e tem como objetivo a produção de sua autonomia e apropriação de seu processo de cuidado. 
Avaliando o processo de trabalho das equipes NASF microrregião de Aquidauana, MS:

Projeto Terapêutico Singular

Dessa forma, o objetivo deste estudo foi analisar a utilização da ferramenta de apoio à atenção o Projeto Terapêutico Singular (PTS) pelas equipes NASF da microrregião de Aquidauana, localizado no Estado de Mato Grosso do Sul.

\section{MATERIAIS E MÉTODO}

Trata-se de uma pesquisa de caráter quantitativo-descritivo, que buscou analisar os dados obtidos por entrevistas com os profissionais do NASF nos anos de 2013 a 2014. Para sua execução, foi selecionada a microrregião de saúde de Aquidauana, em Mato Grosso do Sul, para participar da pesquisa, tendo como critério de seleção os NASF com data de implantação inferior a março de 2011. As visitas aos profissionais de cada um dos NASF selecionados foram agendadas com antecedência, respeitando suas disponibilidades.

Depois de explicitada a proposta e a metodologia empregada neste estudo, assim como a confidencialidade das respostas coletadas, foi solicitado o consentimento livre e esclarecido dos profissionais elencados para entrevista. Em sala reservada, as entrevistas foram realizadas individualmente com esses sujeitos, gravadas e posteriormente transcritas, e estes tiveram a possibilidade de discorrer sobre o tema proposto, sem respostas ou condições pré-fixadas pelos pesquisadores, seguindo um roteiro estruturado de questionamentos norteados para identificar a percepção dos profissionais acerca do tema proposto.

Posteriormente, as ideias centrais foram destacadas caracterizando o discurso coletivo proposto por Lefevre e Lefevre (2014), sobre o Projeto Terapêutico Singular e com base nos discursos, verificamos a utilização dessa ferramenta no NASF. As informações oriundas desse inquérito, incluindo dados racionais e categóricos, foram apresentadas em frequências absolutas. Este estudo foi aprovado pelo Comitê de Ética em Pesquisa da Universidade Católica Dom Bosco, sob Parecer n. 123.226.

\section{RESULTADOS}

Foram entrevistados 15 profissionais de saúde de diferentes equipes NASF da microrregião de Aquidauana, conforme estabelecido com as mi- 
crorregiões de saúde do Estado, prevista na Resolução n. 075/SES/MS de 14 de outubro de 2011, que divide em microrregiões de saúde, uma forma encontrada de descentralizar os serviços do SUS deslocando a gestão do serviço de saúde para os municípios. A microrregião de Aquidauana está inserida dentro da macrorregião de saúde de Campo Grande, que compreende 35 municípios. A microrregião de Aquidauana abrange seis municípios: Anastácio, Aquidauana, Bodoquena, Dois Irmãos do Buriti, Miranda e Nioaque e, de acordo com Censo Demográfico/IBGE de 2010, possui um total de 128.250/habitantes, porém Aquidauana possui o maior porte populacional, com 45.781/habitantes. A situação de saúde da microrregião conta com Hospital Regional, Unidades Básicas de Saúde, que estão inseridas no Programa Estratégia da Saúde da Família (ESF), e outras redes de apoio em saúde, porém somente os municípios de Aquidauana, Anastácio e Dois Irmãos do Buriti haviam implantado, até o momento, o NASF atuando de forma integrada e em conjunto com as equipes Saúde da Família.

Para entender a realidade e como constitui o processo de trabalho dos Núcleos de Apoio à Saúde da Família, é imprescindível saber que estes não constituem porta de entrada do sistema de saúde e se classificam em duas modalidades, o NASF 1 que é composto por no mínimo cinco profissionais com formação universitária e que deve atender no mínimo de oito e um máximo de 20 equipes da Saúde da Família; e o NASF 2, que deve ter no mínimo três profissionais e se vincular a no mínimo três equipes da Saúde da Família. Os municípios participantes da entrevista formavam equipes multiprofissionais, cujas categorias variaram entre assistente social, educador físico, farmacêutico, fisioterapeuta, fonoaudiólogo, nutricionista e psicólogo. Quanto ao perfil dos profissionais entrevistados nos NASF, doze eram do gênero feminino e somente três do gênero masculino, caracterizando um cenário feminino nessa microrregião assistencial de saúde do Estado.

Pensando nas equipes e nas novas práticas, o trabalho em equipe e multiprofissional deve ultrapassar a fragmentação do conhecimento e das especificidades profissionais com o propósito de construir uma prática de cuidado articulada e integrada, uma vez que só dessa forma é que se consegue atender as reais necessidades das pessoas (BOCCARDO et al., 2011). No NASF, cada profissional deve envolver-se com o trabalho através 
Avaliando o processo de trabalho das equipes NASF microrregião de Aquidauana, MS: Projeto Terapêutico Singular

da sua especialidade e todos devem se comprometer com as propostas de promoção da saúde integral, dado que é insatisfatório pensar no indivíduo de forma fragmentada, por áreas de estudo no campo da saúde (OLIVEIRA, 2008).

Outro fator em que é preciso ter atenção é o tempo de atuação do profissional, pois é notável que o tempo de serviço contribui para a meIhoria do processo de trabalho, do desempenho coletivo e individual e, consequentemente, da melhoria da produtividade (RAIMUNDI et al., 2015). Neste estudo, observou-se que esse tempo variou entre alguns profissionais, o qual foi menos de um mês a 72 meses de exercício no NASF, com mediana de nove meses. Nesse contexto, o tempo passa a ter uma afinidade estreita com o trabalho, tornando-se um dos recursos fundamentais de uma organização.

É necessário correlacionar se, nesse período em que está inserido neste campo de trabalho, o profissional esteja passando por atualizações e capacitação para atender a demanda específica que o NASF possui; portanto foi investigado se, ao entrar para a equipe do NASF e durante o tempo de trabalho, o profissional recebeu alguma capacitação ou treinamento dentro da sua área de atuação. Dos 15 profissionais, oito profissionais haviam realizado atualização durante a atuação no NASF, e seis não haviam concluído alguma capacitação até o momento da entrevista no decorrer do trabalho diário. Porém, antes de exercer as atividades dentro do NASF, nenhum dos entrevistados havia passado por educação permanente para trabalhar em consonância com as diretrizes do NASF.

É importante entender que a educação permanente é imprescindível para o fortalecimento das práticas diárias do serviço, apresenta-se como estratégia de reorganização e desenvolvimento dos serviços de saúde, a partir da análise de situações concretas, objetivando mudanças de valores e conceitos e a transformação das práticas dos serviços de saúde (MERHY, 2005).

Dentro da equipe NASF, o principal recurso de trabalho ou sua principal tecnologia de gestão é o apoio matricial que se complementa com o processo de trabalho, e que tem como escopo oferecer tanto retaguarda assistencial quanto amparo técnico-pedagógico às equipes de referência (CUNHA; CAMPOS, 2011). 
Segundo Campos e Domitti (2007, p. 400), "a equipe e os profissionais de referência são aqueles que têm a responsabilidade pela condução e coordenação de um caso individual ou comunitário, responsabilizando-se pela atenção ao longo do tempo, isto é, de maneira longitudinal, à semelhança do preconizado para equipes de saúde da família na atenção básica."

\section{DISCUSSÃO}

É importante compreender, como Nascimento e Oliveira (2010, p. 93) ressaltam, que o momento de formação desses profissionais deve ser discutido e sugere que, "para o desenvolvimento do trabalho interdisciplinar nos moldes em que o NASF propõe, é preciso uma revisão crítica acerca dos processos educativos e formativos desenvolvidos pelo Ensino Superior". O NASF propõe repensar a forma como vêm sendo executadas as práticas em saúde. A transformação da formação e das práticas é um desafio a ser superado em várias instâncias, pois implica mudanças de paradigmas já estruturados nos serviços, nas instituições de ensino e nas relações interpessoais.

Para se trabalhar associando e executando as diretrizes da atenção primária à saúde, o NASF deve privilegiar o atendimento compartilhado, interdisciplinar e intersetorial, com recíproca de saberes, responsabilidades mútuas e capacitação, possibilitando a estruturação do conhecimento e de experiências a todos os envolvidos (OLIVEIRA, 2008). A organização da equipe com base no conceito de "referência" concede o atendimento diversificado e singularizado das demandas dos usuários, a melhoria da acessibilidade aos serviços, profissionais e recursos e a incumbência de todos no processo do cuidado, na medida em que compartilha e descentraliza o atendimento clínico (BOCCARDO et al., 2011).

Além disso, esse processo propõe atingir mudanças sustentáveis, no decorrer do tempo, e a diminuição da dependência exclusiva da população aos serviços de saúde por meio da ativação de recursos exteriores a eles (BOCCARDO et al., 2011). Merhy (2005), salienta que o Projeto Terapêutico Singular (PTS) deve ser uma ferramenta que responda às demandas objetivas e subjetivas dos usuários e tem como propósito a produção de sua autonomia e apoderamento de seu processo de cuidado. Portanto o PTS é um 
Avaliando o processo de trabalho das equipes NASF microrregião de Aquidauana, MS: Projeto Terapêutico Singular

processo no qual todas as opiniões são importantes para ajudar a entender o sujeito ou família com alguma demanda de cuidado em saúde, definindo propostas de ações e estratégias para o cuidado em saúde.

Situações conflitantes dentro do serviço e, muitas vezes, ações que não são resolutivas são visíveis nos serviços de saúde, pois "apresentam dificuldades de acesso da população, fragilidades na criação de vínculos e responsabilizações, bem como uma prática marcada pela frieza dos profissionais e pouca participação da população" (OLIVEIRA; ROCHA; CUTOLO, 2012, p. 575).

Alguns autores evidenciam esse processo de trabalho conflitante:

Em seu processo de trabalho, os profissionais do NASF deparam-se cotidianamente com diversas situações geradoras de estresse e sofrimento, como a dificuldade de aceitação do modelo de atenção proposto pelas equipes da ESF, gestores e usuários, a compreensão do papel de "apoio", o uso de novas tecnologias de cuidado em saúde e o despreparo dos profissionais (ESF e NASF) para atuar em equipe na perspectiva da interdisciplinaridade. (LEITE; NASCIMENTO; OLIVEIRA, 2014, p.508).

Acerca da percepção e aplicabilidade da ferramenta tecnológica de apoio à atenção, o Projeto Terapêutico Singular, oito entrevistados demonstraram o conhecimento sobre este, clareza ao expressar suas ideias e entendimento a respeito do recurso terapêutico, como também compreenderam a necessidade de a equipe compartilhar e apoiar as práticas em saúde nos territórios sob responsabilidade das equipes de Saúde da Família e, com base nos profissionais que souberam explicar do que se tratava a ferramenta, estes exemplificaram sobre o processo no cotidiano da equipe de forma prática, aplicando de acordo com a demanda e salientando a importância desse recurso na organização do trabalho e dos resultados alcançados com a empregabilidade da ferramenta.

Contudo sete profissionais não reconheceram a utilização da ferramenta PTS na prática profissional e explicaram conceitos que não se caracterizam com a ferramenta, como são apresentados e contrários aos princípios doutrinários do SUS, sendo possível notar que não há correta utilização e aplicação no cotidiano do serviço. Consequentemente, o profissional do NASF que desconhece a ferramenta, não atende a demanda corretamente 
da equipe de referência e a necessidade de cuidado em saúde e, desse modo, não há definição de propostas de ações em saúde e integralidade do cuidado do sujeito ou família.

Outro aspecto importante que dever ser reforçado, não somente o conhecimento e a aplicabilidade da ferramenta, mas a construção do projeto terapêutico singular deve ser alicerçado nas tecnologias leves em saúde (acolhimento, vínculo, corresponsabilização e autonomia), que favorecem a adesão do usuário/família ao tratamento, de forma que se construa de forma integral o cuidado em saúde (PINTO et al., 2011).

Nesse sentido, Panizzi (2017, p. 157) ressalta a importância do conhecimento para a mudança do processo de trabalho em saúde:

O trabalho do NASF deve procurar impactar, para além do conhecimento, a forma como a própria EqSF significa o trabalho e o cuidado no cotidiano. Sendo assim, é fundamental um processo pedagógico, que impacte o plano da cognição e também da subjetivação, isto é, forme novas subjetividades, que representam um dos fatores de mudança dos sujeitos e, por consequência, do processo de trabalho.

Dessa maneira, a educação permanente em saúde deve ser tratada como aliada ao profissional da saúde, dado que a educação alia a teoria à prática, observando as reais necessidades dos profissionais e população atendida. Santos et al. (2017, p. 61) assim corroboram, ao dizer que "a carência de profissionais preparados para a abordagem integral do usuário apresenta-se como um dificultador na efetivação das ações do NASF, bem como da ESF."

Portanto é imprescindível esclarecer e assimilar a utilização da ferramenta e como devem ser consideradas as tecnologias de apoio à atenção:

Assim, os projetos terapêuticos são elaborados com base nas necessidades de saúde de cada usuário, levando em consideração seu modo de compreender a vida, suas subjetividades e singularidades, configurando-se numa interação democrática e horizontal entre os atores envolvidos no processo de cuidar (trabalhadores/usuário/ família), alicerçados pela utilização das tecnologias das relações que propõe novos fluxos e circuitos dentro do sistema, seguindo os movimentos dos usuários e a incorporação de novas tecnologias de trabalho e de gestão. (PINTO et al., 2011 p. 501) 
Avaliando o processo de trabalho das equipes NASF microrregião de Aquidauana, MS: Projeto Terapêutico Singular

Contudo Barros (2009) aponta que há obstáculos, que são observados para o desenvolvimento do Projeto Terapêutico Singular, como: a dificuldade da equipe em detectar a base teórica de sua prática; a sobrecarga de responsabilidade assistencial motivada pela alta demanda; a dinâmica proposta para as reuniões; e a carência de qualificação das equipes. E foi revelado nos discursos dos profissionais do NASF que há uma alta demanda em atendimentos clínicos, individualizados e especializados, uma vez que a rede de serviços de saúde não capta essa necessidade em saúde, e também por desconhecer por parte dos profissionais da Atenção Básica e da gestão qual a real finalidade do NASF. Logo, um dos principais desafios também vivenciados pelos profissionais do NASF é fazer uso contínuo do matriciamento e incorporá-lo efetivamente às ações cotidianas junto às equipes de Saúde da Família.

Realizar o apoio matricial implica trocar ideias e informações, ajustar expectativas, pactuar decisões. Campos e Domitti (2007) entendem que o apoio matricial provoca uma reformulação na organização dos serviços, de forma que as áreas especializadas, outrora verticais, possam oferecer apoio técnico pedagógico horizontal às equipes da atenção primária. E, para a utilização dessa estratégia, é necessário mudanças ou mesmo uma transformação global na maneira como se organizam e funcionam os serviços de saúde (BARROS et al., 2015).

Dessa maneira, o PTS é uma ferramenta de apoio à atenção e soma valores importantes como um elemento do processo do cuidado que, a partir do acolhimento reorganizando e o processo de trabalho, pode ser compreendido e abordado de formas diversas, no contexto de um mesmo serviço (BOCCARDO et al., 2011). Minimizar a importância e necessidade das ferramentas tecnológicas, tanto de apoio a gestão, quanto de apoio à atenção, provoca uma série de prejuízos no desenvolvimento das ações de promoção a saúde para a população atendida, deixando de captar o sujeito singular e suas subjetividades.

Não havendo essa transição, continua-se a trabalhar no modelo antigo contribuindo para o enfraquecimento do modelo de atenção primária à saúde, centrado na atuação apenas quando o usuário está com queixas pontuais ou adoecimento, e a consequência dessa atenção visando à prática 
curativa, está na medicalização excessiva, altos custos, baixa autonomia dos usuários, redução da capacidade de atuação dos profissionais dos serviços de saúde e dificuldade de vinculação com outros serviços (ESMERALDO et al., 2017).

LINASSI (2013, p. 432) entende essa transição do modelo:

Acredita-se que esta transição é lenta, pois a clínica tradicional (atuação reducionista) está enraizada (com vícios e mazelas) no modo de fazer saúde no Brasil, o qual é, por diversas vezes, muito mais doentio do que saudável. A mudança de atitude necessária para a superação desta clínica degradada não se restringe somente à atuação dos profissionais que estão inseridos no Sistema Único de Saúde (SUS), mas também deve ser uma mudança buscada e experienciada nas instituições acadêmicas, formadoras de futuros profissionais da saúde.

É imprescindível gerar uma comunicação interativa e inovadora, que se co-responsabiliza e compartilha as necessidades entre os diversos serviços em diferentes níveis de atenção, pois ainda é visível a independência do trabalho em saúde, tanto na referência quanto na contra-referência, o que prejudica o avanço e efetividade das ferramentas tecnológicas de assistência. Portanto a qualidade da atenção e a satisfação dos trabalhadores também dependerão de como a gestão facilita essa interação e apoio entre profissionais e serviços de saúde. A produção de saúde desejada através do PTS é aquela que deve ser coproduzida por todos, porque ninguém produz saúde sozinho. Trabalhadores de saúde (com apoio da gestão, mas sem intervenção direta) e usuários devem movimentar-se ousando pensar em um novo modo de fazer, promover e produzir saúde.

\section{CONSIDERAÇÕES FINAIS}

Este estudo corrobora com a necessidade do profissional da equipe NASF conhecer e utilizar, de forma efetiva, as ferramentas tecnológicas do NASF, especificamente o Projeto Terapêutico Singular, que favorece o trabaIho interdisciplinar e uma terapêutica centrada no usuário, com qualidade, resolubilidade e equidade. O grupo de profissionais que pactuou viver essa experiência de apontar seu conhecimento sobre Projeto Terapêutico 
Avaliando o processo de trabalho das equipes NASF microrregião de Aquidauana, MS:

Projeto Terapêutico Singular

Singular concluiu que, mesmo enfrentando tantas dificuldades e entraves, os resultados foram significativos. O sujeito é assistido de modo integral, reabilitando e promovendo a saúde de forma holística. Portanto o PTS deve ser valorizado no que se trata de promoção à saúde, já que produz saúde através dos impactos gerados de suas ações que atuam diretamente sobre os condicionantes e determinantes sociais da saúde da população. Nesse contexto, a gestão dos serviços é indubitavelmente necessária para o funcionamento do NASF.

\section{REFERÊNCIAS}

BARROS, Juliana de Oliveira. A construção de projetos terapêuticos no campo da saúde mental: apontamentos acerca das novas tecnologias de cuidado. 2009. Dissertação (Mestrado em Ciências) - Universidade de São Paulo, São Paulo, SP, 2009.

BARROS, Juliana de Oliveira et al. Estratégia do apoio matricial: a experiência de duas equipes do Núcleo de a poio à saúde da família (NASF) da cidade de São Paulo, Brasil. Ciência \& Saúde Coletiva, Rio de Janeiro, v. 20, n. 9, p. 2847-56, 2015.

BRASIL. Ministério da Saúde. Secretaria de Atenção à Saúde. Departamento de Atenção Básica. Diretrizes do NASF: núcleo de apoio à saúde da família. Brasília: Ministério da Saúde, 2010. 157p. (Série A. Normas e Manuais Técnicos/Cadernos de Atenção Básica, n. 27).

. Ministério da Saúde. Portaria n. 154, de 24 de janeiro de 2008. Cria os Núcleos de Apoio à Saúde da Família - NASF. Disponível em: < http://bvsms.saude. gov.br/bvs/saudelegis/gm/2008/prt0154_24_01_2008.html>.

BOCCARDO, Andréa C. S. et al. O projeto terapêutico singular como estratégia de organização do cuidado nos serviços de saúde mental. Revista de Terapia Ocupacional da Universidade de São Paulo, São Paulo, v. 22, n. 1, p. 85-92, jan./ abr. 2011.

CAMPOS, Gastão Wagner de Sousa; DOMITTI, Ana Carla. Apoio matricial e equipe de referência: uma metodologia para gestão do trabalho interdisciplinar em saúde. Cadernos de Saúde Pública, Rio de Janeiro, v. 23, n. 2, p. 399-407, fev. 2007.

CUNHA, Gustavo T.; CAMPOS, Gastão W. S. Apoio matricial e atenção primária em saúde. Saúde e Sociedade. São Paulo, v. 20, n. 4, p. 961-70, 2011.

ESMERALDO, Geordany Rose de Oliveira Viana et al. Tensão entre modelo biomédico e estratégia saúde da família: percepções dos trabalhadores de saúde. Revista de APS, Juiz de Fora, v. 20, n. 1, 2017. 
FERTONANI, Hosanna Pattrig et al. Modelo assistencial em saúde: conceitos e desafios para a atenção básica brasileira. Ciência \& Saúde Coletiva, v. 20, n. 6, p. 1869-78, 2015.

GONÇALVES, Rita Maria de Abreu et al. Estudo do trabalho em Núcleos de Apoio à Saúde da Família (NASF), São Paulo, Brasil. Revista Brasileira de Saúde Ocupacional, v. 40, n. 131, 2015.

INSTITUTO BRASILEIRO DE GEOGRAFIA E ESTATÍSTICA (IBGE). Censo Demográfico 2010. IBGE, 2010.

LEFEVRE, Fernando; LEFEVRE, Ana Maria Cavalcanti . Discurso do sujeito coletivo: representações sociais e intervenções comunicativas. Texto Contexto Enfermagem, Florianópolis, v. 23, n. 2, p. 502-7, abr. 2014. Disponível em: <http://www.scielo. br/pdf/tce/v23n2/pt_0104-0707-tce-23-02-00502.pdf>. Acesso em: 14 out. 2017.

LEITE, Denise Fernandes; NASCIMENTO, Débora Dupas Gonçalves do; OLIVEIRA, Maria Amélia de Campos. Qualidade de vida no trabalho de profissionais do NASF no município de São Paulo. Physis: Revista de Saúde Coletiva, v. 24, n. 2, p. 50725, 2014.

LINASSI, Jordana et al. Projeto terapêutico singular: vivenciando uma experiência de implementação. Revista Contexto \& Saúde, v. 11, n. 20, p. 425-34, 2013.

MATO GROSSO DO SUL. Secretária de Estado de Saúde. Resolução n. 075/SES/MS. Aprova as decisões da Comissão Intergestores Bipartite Estadual. Diário Oficial n. 8.061, Campo Grande, 14 de novembro de 2011, p. 14.

MERHY, Emerson Elias; FRANCO, Túlio Batista. Por uma Composição Técnica do Trabalho em saúde centrada no campo relacional e nas tecnologias leves. Apontando mudanças para os modelos tecno-assistenciais. Saúde em Debate, Rio de Janeiro, v. 27, n. 65, p. 316-23, 2003.

MERHY, Emerson Elias. O desafio que a educação permanente tem em si: a pedagogia da implicação. Interface (Botucatu), Botucatu, v. 9, n. 16, p. 172-4, set./fev. 2005.

MORORÓ, Martha E. M. L. Cartografias, desafios e potencialidades na construção de projeto terapêutico em Centro de Atenção Psicossocial - CAPS III. 2010. $130 f$. Dissertação (Mestrado em Enfermagem) - Universidade de São Paulo, São Paulo, 2010.

NASCIMENTO, Débora D. G.; OLIVEIRA, Maria A. C. Reflexões sobre as competências profissionais para o processo de trabalho nos Núcleos de Apoio à Saúde da Família. O Mundo da Saúde, São Paulo, v. 34, v. 1, p. 92-6, 2010.

OLIVEIRA, Gustavo N. O projeto terapêutico e a mudança nos modos de produzir saúde. São Paulo: Hucitec, 2008. 
OLIVEIRA, Inajara C; ROCHA, Renata M; CUTOLO, Luiz R. A. Algumas palavras sobre o NASF: relatando uma experiência acadêmica. Revista Brasileira de Educação Médica, Rio de Janeiro, v. 36, n. 4, p. 574-80, dez. 2012.

PANIZZI, Mirvaine et al. Reestruturação produtiva na saúde: atuação e desafios do Núcleo de Apoio à Saúde da Família. Saúde em Debate, Rio de Janeiro, v. 41, n. 112, p. 155-70, 2017.

PINTO, Diego Muniz et al . Projeto terapêutico singular na produção do cuidado integral: uma construção coletiva. Texto \& Contexto - Enfermagem, Florianópolis, v. 20, n. 3, p. 493-502, jul./set. 2011.

RAIMUNDI, Daniele Merisio et al. A prática de uma clínica da família na perspectiva dos enfermeiros do serviço. Investigação Qualitativa em Saúde, Aracaju, v. 1, p. 579-84, ago. 2015. Disponível em: <http://www.proceedings.ciaiq.org/index.php/ ciaiq2015/article/view/131/127>. Acesso em: 14 out. 2017.

SANTOS, Maria Clara, et al. Processo de Trabalho do Núcleo de apoio à Saúde da Família (NASF): importância da Qualificação Profissional. Saúde \& Transformação Social, Florianópolis, v. 8, n. 2, p. 60-9, maio/ago. 2017. 
